\title{
Barreras, facilitadores y estrategias que influyen en la participación femenina en juntas directivas de federaciones deportivas chilenas Barriers, facilitators and strategies that influence women's participation on boards of directors of Chilean sports federations
}

*Gerard Foppiano-Vilo, **Carlos Matus-Castillo, ***Miguel Cornejo-Améstica

*Universidad Federal de Paraná (Brasil), **Universidad Católica de la Santísima Concepción (Chile), ***Universidad de Concepción (Chile)

Resumen. La brecha de género sobre la participación femenina en puestos de toma de decisiones está evidenciada en las organizaciones deportivas. Las juntas directivas de las Federaciones Deportivas chilenas no están ajenas a esta situación. Por ello, esta investigación tuvo como objetivo determinar barreras, facilitadores y estrategias que influyen en la participación y desarrollo de las mujeres en cargos directivos en las Federaciones Deportivas de Chile. El método fue de tipo cualitativo con enfoque descriptivo y, la técnica fue la entrevista personal mediante aplicación de un guion semi-estructurado de preguntas. Durante octubre y noviembre de 2020 se desarrollaron entrevistas virtuales a mujeres que formaban parte de juntas directivas de federaciones deportivas. El análisis se realizó mediante categorías de análisis y codificaciones empleando el software Atlas. Ti. Las barreras identificadas se asocian a situaciones familiares tales como el cuidado de miembros de la familia. También se reconoce la existencia de prejuicios asociados a estereotipos de género en las funciones que se desempeñan; subestimación de las opiniones femeninas y desarrollo de los cargos en ambientes masculinizados que invisibilizan a las mujeres. Como agentes facilitadores se identifica el soporte familiar; el tener una profesión, experiencia laboral y capacitación en gestión deportiva, junto con algunos aspectos de la personalidad de las dirigentes. Como estrategias se releva la necesidad de un cambio cultural al interior de las federaciones en torno a la participación femenina, implementar cuotas de participación por género y desarrollar programas de capacitación en liderazgo para las mujeres.

Palabras clave: brecha de género; mujer; participación; organizaciones deportivas; junta directiva; dirigente.

\begin{abstract}
The gender gap in female participation in decision-making positions is evident in sports organizations. The boards of directors of Chilean sports federations are no strangers to this situation. Therefore, the objective of this research was to determine barriers, facilitators and strategies that influence the participation and development of women in managerial positions in Chilean Sports Federations. The method was qualitative with a descriptive approach and the technique was the personal interview through the application of a semi-structured script of questions. During October and November 2020, virtual interviews were conducted with women who were members of the boards of directors of sports federations. The analysis was carried out through categories of analysis and coding using Atlas. Ti. The barriers identified are associated with family situations such as caring for family members. The existence of prejudices associated with gender stereotypes in the functions performed, underestimation of women's opinions and the development of positions in masculinized environments that make women invisible are also recognized. Family support is identified as facilitating agents, having a profession, work experience and training in sports management, together with some aspects of the leaders' personalities. As strategies, the need for a cultural change within the federations regarding women's participation, the implementation of participation quotas by gender and the development of leadership training programs for women were identified.
\end{abstract}

Key words: gender gap; woman; participation; sports organisations; board of directors; leader.

\section{Introducción}

Las mujeres y niñas representan a la mitad de la población mundial, por tanto, la mitad de su potencial, es decir, deberían tener una representación equilibrada en todas las instancias de la sociedad. Sin embargo, la desigualdad de género existe, persiste y provoca el es-

Fecha recepción: 26-06-21. Fecha de aceptación: 05-10-21

Carlos Matus-Castillo

cmatus@ucsc.cl tancamiento del progreso social (ONU Mujeres, 2021; ONU, s. f.). Si bien es cierto, y como reconoce la ONU, han existido avances en las últimas décadas y, actualmente más niñas participan de procesos de escolarización, los marcos legales de los países se están reformando para fomentar la igualdad, existe más participación de mujeres en cargos políticos y de liderazgo (ONU, 2015), aun los desafíos son elevados, a fin de lograr la igualdad y equidad de género. Un ejemplo de esta situación son los mínimos avances reportados por 
el informe: Women in the boardroom: A global perspective -6 th edition de la consultora Deloitte (2019), la cual evidencia la presencia de las mujeres en cargos directivos de empresas privadas a nivel mundial. Los datos del año 2018 evidenciaron que solo el 16.9\% de puestos en las juntas directivas a nivel mundial estaba cubierto por mujeres, mostrando un incremento de solo el 1.9\% en comparación al año 2016 (15\%). En el caso particular de Chile, el mismo informe reportó que el año 2018 las mujeres en cargos directivos representaban solo el 7.7\% y el año 2016 el 6.5\%. En este contexto, las organizaciones deportivas no escapan a la subrepresentación femenina en puestos de liderazgo (Acosta \& Carpenter, 2014; Smith \& Wrynn, 2013), tanto a nivel voluntario como profesional (Claringbould \& Knoppers, 2008), incluido el deporte olímpico (Smith \& Wrynn, 2013). En Chile, la participación femenina en puestos directivos (presidenta, vicepresidenta, secretaria, tesorera y directora) de las 56 Federaciones Deportivas afiliadas al Comité Olímpico de Chile (COCH) en el año 2020 ascendía solo al 11.5\% de 253 cargos, los que se distribuyen de la siguiente forma: 0.8\% de Presidentas; 2\% Vicepresidentas; 2\% de Tesoreras; $3.2 \%$ de Secretarias y 3.6\% de Directoras $(\mathrm{COCH}, 2020)$. Esta infrarrepresentación puede observarse también en otros contextos, tal como reportan Adriaanse y Claringbould (2016) quienes analizaron los porcentajes de representación femenina en organizaciones deportivas de 45 países de cinco continentes; observando que en promedio la presencia de mujeres dirigentes solo ascendía al 19.7\%. Además, esta situación se ha pesquisado de forma particular en Brasil (Zanatta et al., 2018), en Siria (Megheirkouni, 2014), en Estados Unidos (Gaston et al., 2020), Australia (Adriaanse \& Schofield, 2014) y en Europa en su conjunto (Adriaanse, 2019).

Esta baja presencia podría ser explicada y comprendida a través del análisis de los estereotipos de género, como la teoría del Techo de Cristal, la Teoría Homosocial de Kanter o El Régimen de Género de Connell. En este sentido comprenderemos los estereotipos de género como la asignación social de roles y responsabilidades diferenciadas, que condicionan opciones, hábitos y desempeños, condicionando lo femenino y lo masculino (Ávila \& Corvalán, 2016). Los estereotipos incorporan muchos componentes, tales como la personalidad, las funciones familiares, el empleo, las características físicas y la orientación sexual (De Lemus \& Moya, 2004). Para Menéndez (2020) los estereotipos de género sostienen códigos de conducta que determinan e imponen los límites desde los cuales se piensa el género; siendo un paradigma que, aparentando universalidad, constituye un mandato normativo unidireccional y muy resistente al cambio; lo que ha favorecido a la dominación de los varones en el ámbito deportivo. Moragas (2014) indica que los estereotipos tienen un carácter prescriptivo, puesto que indican las conductas adecuadas para las mujeres o para los hombres en función de su género; por tanto, condicionan sus comportamientos en todas las esferas de la vida, incluyendo el ámbito deportivo. Sobre este último punto, Menéndez (2020) sostiene que el acceso de las mujeres al deporte se ha realizado de forma tardía y sin eliminar los estereotipos de género imperantes en la actividad física, los cuales le otorgan una característica intrínsecamente masculina. Un factor prematuro que potenciaría los estereotipos de género y la masculinización del deporte radicaría, por ejemplo, en el uso significativamente mayoritario de imágenes masculinas en los textos de educación física escolar, lo cual reduce la visibilidad de modelos deportivos femeninos (Ruiz-Rabadán \& Moya-Mata, 2020).

Por otro lado, la teoría del Techo de Cristal (Glass Ceiling) es acuñada por Hymowitz y Schelhardt (1986) para referirse a barreras artificiales e invisibles que impiden a personas cualificadas avanzar en las organizaciones hacia posiciones más altas (Baumgartner \& Schneider, 2010), debido frecuentemente a las prácticas conscientes e inconscientes de discriminación y desigualdad en las organizaciones laborales. Barberá et al. (2002) destacan al Techo de Cristal como un factor significativo en cualquier sector laboral analizado, incluso los más feminizados; donde la proporción de mujeres baja en la medida en que sube la jerarquía de la organización, de modo que su presencia ocupando posiciones de poder es mínima. El Techo de Cristal también está presente en la dirección del deporte; siendo las organizaciones deportivas reconocidas como instituciones donde a menudo no se observan mujeres en las cúpulas de las juntas directivas. Es posible apreciar que mientras más alto es el puesto, es menos probable encontrar mujeres, lo que se condice con lo expuesto por Marugán (2017) quien argumenta que, en el funcionamiento interno de las organizaciones deportivas, estas están impregnadas de prácticas discriminatorias, muchas de las cuales no son fáciles de observar. En efecto, la investigación de Adriaanse y Claringbould (2016), que analiza las juntas directivas de las federaciones deportivas de 45 países, determina que sólo el $10.8 \%$ de ellas son presididas por mujeres; mientras que el de directoras ejecutivas asciende a un $16.3 \%$. Estos datos dejan en- 
trever que el fenómeno delTecho de Cristal afecta a las organizaciones deportivas globalmente. Oakley (2000) explica que las mujeres no llegan a puestos más altos por: a) oportunidades profesionales inadecuadas, b) estereotipos basados en el género, c) existencia de redes masculinas y d) el simbolismo. El mismo autor señala otras barreras que incluyen las diferencias entre estilos de liderazgo femenino y el tipo de liderazgo esperado por los líderes de las organizaciones. En esta misma línea, Parzinger et al. (2001) indican como posibles barreras para la mujer: factores asociados al nivel educativo y factores familiares, las culturas corporativas y factores sociológicos con influencia masculina. Otras razones de la subrepresentación estarían relacionadas con la «naturalidad» percibida de los hombres que ocupan esos puestos (Shaw \& Hoeber, 2003). Así, el Techo de Cristal describe como la carrera laboral o dirigencial femenina se estanca en un nivel jerárquico medio o bajo, sin posibilidades de alcanzar puestos de poder para la toma de decisiones, simplemente por ser mujeres o por preconceptos raciales, siendo esto una evidente discriminación.

Otra perspectiva teórica que surge es la propuesta de Kanter (1977) sobre la Reproducción Homosocial, la cual propone que, en una estructura organizacional dominada por hombres, estos reciben mayores oportunidades y poder debido a su representación mayoritaria. Kanter indica que los agentes masculinos guardan poder y privilegios para aquellos que son como ellos, esto quiere decir que es más probable que hombres contraten o elijan a otros hombres similares a ellos, independiente de sus cualidades, realizando sus elecciones sobre la base de principios sociales y morales en los que fueron educados, generalmente en un régimen donde el poder social es ejercido por los hombres. Sumado a lo anterior, Moragas (2014) indica la existencia de «redes masculinas» que se desarrollan en centros sociales exclusivos para hombres (clubes sociales, campos de golf, etc.), donde las mujeres tradicionalmente son excluidas y no pueden participar, lo cual dificulta el acceso de ellas a información para la toma de decisiones, siendo esto una desventaja para competir por un puesto directivo. La Reproducción Homosocial tiene como resultados una menor proporción de mujeres en puestos gerenciales, menos modelos femeninos a seguir, perpetuación de los estereotipos de género y segregación sexual ocupacional.

Otro punto de análisis proviene desde las relaciones sociales, en este marco, Connell (2009) propone el Régimen de Género, manifestando que las relaciones de género son establecidas a través de la práctica social. Connell plantea cuatro dimensiones principales en la articulación género-práctica social: a) Producción (trabajo organizado por género), b) Poder (cómo y quién ejerce el control), c) Emociones (influencian las relaciones) y d) Simbolismo (creencias, actitudes y valores). La interacción y combinación de esas dimensiones más el patrón de relaciones de género resultante forma lo que la autora llama el «Régimen de Género». La normalización de este «Régimen» ha sido implantado en la sociedad por diversos agentes socializadores, tales como la familia, el estado, la iglesia, la escuela o el deporte. De esta forma, estos agentes exigen a las mujeres cumplir con sus papeles «tradicionales» como hija, esposa y madre; impidiendo su libre desarrollo en actividades laborales, estudios o la dirección de organizaciones, puesto que interfieren con sus «obligaciones naturales». De esta forma, las dificultades que enfrentan las mujeres son muchas veces minimizadas, dado que esta misma sociedad considera que formalmente se ha alcanzado la igualdad; puesto que se ha naturalizado la jerarquía entre los sexos (Monforte \& Úbeda-Colomer, 2019). Las dimensiones presentadas por Connell pueden ser complementadas a través de los trabajos de Bourdieu (2000), para quien la fuerza del orden masculino es visible en el hecho donde la visión androcéntrica es impuesta como neutra y no siente la necesidad de anunciarse en discursos capaces de legitimarla. De ahí que el orden social ratifica la dominación masculina a través de la división sexual del trabajo, de las actividades asignadas a cada sexo, los espacios ocupados por hombres y mujeres en la sociedad, entre otras; pudiendo ser esta una de las razones por la cual existe una baja representación de mujeres en las juntas directivas deportivas, pues de acuerdo con las creencias de la sociedad los puestos de liderazgo están reservados para los hombres.

Producto de lo anterior, es que surge la necesidad de conocer la situación de las mujeres en las organizaciones deportivas, respecto a su participación y roles. Como bien señalan Álfaro (2008) y González Tirados (2010), no basta solo con medir el número de niñas/ mujeres que participan de organizaciones, sino también es importante conocer sus realidades y situación sobre la toma de decisiones.

En el ámbito deportivo, una de las principales motivaciones para promover la participación femenina en puestos de toma de decisiones, radica en considerar que las mujeres también son partes interesadas (stakeholders) de las organizaciones, y que su inclusión 
es un talento disponible en las instituciones (Adriaanse \& Claringbould, 2016). Terjesen et al. (2009) explican que la diversidad de género en las juntas directivas contribuye a la eficacia y que la presencia femenina otorga más sensibilidad hacia otras perspectivas. La ONG Women in Sport (2015) señaló que entre los beneficios que aporta la mujer a las organizaciones deportivas están: la variedad y experiencias en gestión; otorgan más progresismo y creatividad; permiten conocer cómo involucrar a más mujeres y niñas en el deporte. Pfister (2010) señala que las mujeres aportan perspectivas o ideas nuevas para la junta directiva, mejorando así la atmósfera de reuniones. Por otro lado, Acosta y Carpenter (2014) manifiestan que la participación femenina implica la transformación de modelos femeninos, derivando en un efecto que promueve el interés de las mujeres por participar de la dirigencia deportiva. El objetivo de este estudio es determinar barreras, facilitadores y estrategias que influyen en la participación y desarrollo de las mujeres en cargos directivos en las Federaciones Deportivas de Chile.

\section{Material y método}

\section{Tipo de estudio}

Investigación cualitativa con enfoque descriptivo, dado que estas se realizan cuando un problema necesita ser explorado, o cuando se necesita una comprensión compleja y detallada de una cuestión (Creswell, 2014). De igual forma, Banks (2010) señala que este tipo de estudios puede ser abordado analizando experiencias de individuos o grupos, examinando interrelaciones y comunicaciones e investigando documentos. En lo referente al enfoque descriptivo, Hernández et al. (2014) indican que estos buscan especificar las propiedades, las características y los perfiles de personas, grupos, comunidades, procesos, objetos o cualquier otro fenómeno que necesite ser analizado con la finalidad de describir las tendencias de un grupo o población, siendo los estudios descriptivos útiles para mostrar las dimensiones de un fenómeno.

\section{Participantes}

Los criterios de inclusión de las dirigentes fueron: pertenecer a la población femenina chilena económicamente activa (18 a 60 años); tener residencia en el país; ser integrante de la junta directiva de una federación deportiva al momento de la entrevista (deporte colectivo o individual); poseer estudios universitarios o técnicos concluidos. Cabe señalar que los cargos de las jun- tas directivas de las federaciones deportivas chilenas son de tipo voluntario y no se les asigna remuneración (sueldo). Finalmente las participantes del estudio fueron seis mujeres adultas y dirigentes de juntas directivas de seis federaciones deportivas chilenas (Comité Olímpico de Chile, 2020); sus edades fluctuaban entre los 35 y 58 años; cinco tenían el cargo de Secretaria y una el de Presidenta; cuatro representaban a deportes individuales y dos a deportes colectivos; cinco tenían uno o más hijos/as; cuatro estaban casadas y dos divorciadas; todas contaban con una formación profesional o técnica; cuatro manifestaron tener un trabajo estable, una señaló que no estaba en esa condición y otra omitió responder esta consulta. El hecho de que fuesen seis las dirigentes entrevistadas, radica en el bajo número de mujeres que existen actualmente en las federaciones deportivas chilenas (11.5\%), dicha situación influyó en la convocatoria, cobertura y en la capacidad de respuesta positiva para participar del estudio. No obstante, las seis entrevistadas representan el $20.7 \%$ del total de mujeres dirigentes de federaciones deportivas chilenas.

\section{Técnica e instrumento}

Se empleó como técnica la entrevista personal cualitativa. Heinemann (2008) indica que esta técnica se utiliza en una fase exploratoria; sobre todo cuando se busca información sobre vivencias subjetivas, procesos, sucesos biográficos y valoraciones de situaciones cotidianas. El mismo autor define la entrevista cualitativa como una entrevista individual, no estandarizada; en la cual, mediante preguntas formuladas en el contexto de la investigación, las personas emiten informaciones útiles para resolver la pregunta de investigación. Con eso, es posible obtener una imagen e ideas propias de los sujetos, permitiendo conocer la realidad que perciben en un determinado escenario. En este estudio la información se recopiló en seis entrevistas cualitativas de tipo semi-estructuradas. Como instrumento se empleó un guion semi-estructurado, el cual fue diseñado por el equipo de investigación en concordancia con los objetivos del estudio, luego se aplicó una entrevista piloto a una dirigente que cumplía con los criterios de inclusión de las participantes, esto permitió revisar y ajustar las preguntas, también la estructura del guion y determinar el tiempo aproximado de aplicación.

Las dimensiones del guion semi-estructurado fueron: 1. Experiencia deportiva (como practicante) y su relación con el cargo directivo; 2. Experiencias dirigenciales en cargos directivos; 3. Elementos facilitadores y barreras para el acceso a cargos directi- 
vos; 4. Elementos que favorecen o dificultan la participación en la toma de decisiones en los puestos de gobernanza; 5. Percepción sobre la participación femenina en puestos directivos. Para el desarrollo de esta investigación y de acuerdo con el objetivo, se han priorizado las opiniones de las dimensiones 1, 3 y 4 .

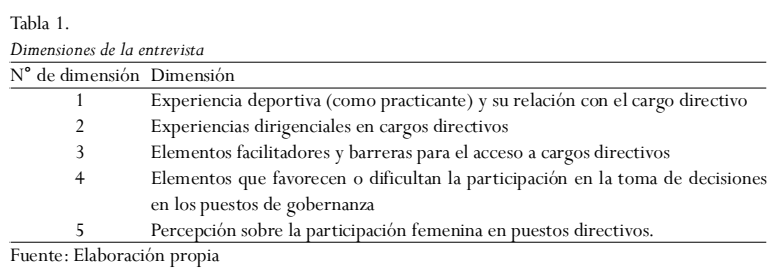

\section{Procedimiento}

Se realizó una revisión y luego una lista de las personas integrantes de los directorios de las federaciones la cual incluía: nombre de la federación, nombre dirigente, género, cargo en el directorio y contacto (correo electrónico y/o teléfono). Posteriormente se realizó un filtro y selección en función de los datos y los criterios de inclusión. Luego se contactó por correo electrónico y/o teléfono a las dirigentes que cumplían los criterios.

En función de la respuesta (positiva, negativa o sin respuesta) se realizó una llamada telefónica para explicar los objetivos de la investigación y coordinar la entrevista. Finalmente fueron seleccionadas seis dirigentes.

Las entrevistas se realizaron durante octubre y noviembre del año 2020 de forma virtual mediante la aplicación de videollamadas Google Meet, la cual permitió grabar las entrevistas, para luego transcribir las declaraciones y analizar la información. La videollamada se empleó debido a dos factores: la situación sanitaria (COVID 19), lo cual impedía desarrollar actividades presenciales y las distancias físicas entre el entrevistador y la entrevistada. La entrevista mediante videollamadas ha sido estudiada y validada por diversos autores (Lo Iacono et al., 2016; Sullivan, 2013), quienes concuerdan en que este mecanismo tiene validez similar a las entrevistas personales y presenciales. Las entrevistas tuvieron una duración promedio de $45.6 \mathrm{mi}$ nutos y las dirigentes firmaron un consentimiento informado para el uso de la información de manera anónima.

\section{Análisis de los datos}

El análisis de las entrevistas se realizó mediante el establecimiento de tres categorías de análisis: Categoría 1: Barreras para el acceso y participación en cargos directivos; Categoría 2: Facilitadores para el acceso y participación en cargos directivos; Categoría 3: Estrategias y propuestas para el acceso y participación en cargos directivos. A cada una de estas categorías se le asignó una codificación conceptual para su identificación. Los códigos son una abreviación que es aplicada a frases o parágrafos que se generaron en las transcripciones de las entrevistas. A partir del objetivo de la investigación se pesquisaron situaciones recurrentes al interior de los discursos. Glaser y Strauss citados por Flores (2009) indican que la codificación es un procedimiento mediante el cual son identificados los conceptos y se descubren las propiedades y dimensiones de la información. Una vez creadas las categorías, las entrevistas se analizaron mediante códigos, empleando el software ATLAS.Ti. El programa utiliza los códigos creados por el investigador, permitiendo ordenar, clasificar y seleccionar la información.

\section{Resultados}

A continuación, se presentan los resultados de la investigación clasificados en los tres ejes que sustentan el estudio: barreras, facilitadores y estrategias que permiten el acceso y desarrollo en los cargos directivos. La tabla 2 muestra las codificaciones más recurrentes identificadas en las declaraciones de las entrevistadas en cada categoría. Posteriormente se muestran las citas más relevantes aportadas por las dirigentes. Se han alterado los nombres de las entrevistadas para resguardar su anonimato.

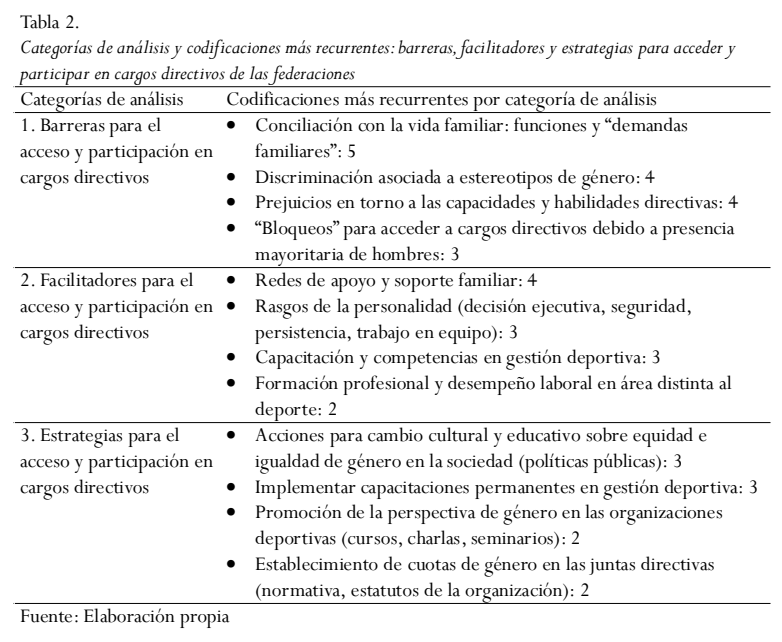

\section{Barreras}

Las barreras con relación al acceso y participación en cargos directivos se generan en un contexto en el cual la totalidad de las juntas directivas están integradas mayoritariamente por hombres. En este ambiente, las 
dirigentes manifiestan que su participación se ve dificultada por aspectos y demandas familiares, tales como la maternidad, el cuidado de los hijos y las funciones domésticas, lo que les impide contar con el tiempo suficiente para participar en las organizaciones. Se observa como una barrera relevante la conciliación de la vida familiar con las actividades de la federación.

"... a un hombre nunca le van a preguntar: tú que eres candidato a dirigente y tienes 3 o 4 hijos, ¿cómo vas a compatibilizar tu trabajo, los hijos y la casa? No existe esa pregunta en el mundo masculino. Pero a mí me tocó enfrentarla y fue chocante, fue ¿cómo me preguntan eso?, estamos en el siglo XXI. Creo que el prejuicio social todavía está muy arraigado en Chile, y no solamente en el deporte, en lo laboral te discriminan por ser mamá.Y es que todo ese mundo que vivimos nosotras (la familia), no fuese un mundo que pertenece a los hombres...» (Fernanda, 35 años, 3 hijos, divorciada).

"Y eso fue algo que no quise hacer (renunciar), no quise aumentar esa cifra de mujeres que abandonan cargos porque tu familia, tu entorno y todo te supera» (Carolina, casada).

"... lo he visto en otras compañeras dirigentes con los hijos, por ejemplo, muchas veces tienen el apoyo de las familias, y esposos, y es como: si, te apoyo, pero en el momento real cuando hay que dejar a los hijos para ir a una reunión de la organización te dicen: bueno, yo también tengo cosas que hacer, no los puedo ver. Porque siempre se asume que los niños son responsabilidad de la mujer» (Amelia, 36 años, 1 hijo, divorciada).

Otra barrera identificada es la falta de confianza y prejuicios de parte de directivos varones sobre las capacidades técnicas de sus pares mujeres. Ellos consideran que la condición de ser mujeres implica no tener las mismas capacidades que ellos.

"... se duda mucho de las capacidades de las mujeres, siempre se piensa que un hombre sabe todo, sobre todo acá en Chile... nosotras las mujeres no pasamos del puesto de Secretaria, es muy difícil que, en una federación, en Chile por lo menos, exista una mujer Presidenta; pero cuando estuve en Lima con las demás mujeres de Latinoamérica, no había en ningún país sudamericano una mujer, escasamente llegaban a ser Secretarias» (Camila, 58 años, 1 hijo, casada).

"... yo creo que un fuerte prejuicio sobre las mujeres dirigentes se relaciona con las capacidades que tenemos nosotras, en la poca valoración de poder participar en el mundo masculino de los dirigentes; en como organizamos el quehacer de la casa, el trabajo, los niños, la familia...» (Fernanda, 35 años, 3 hijos, divorciada).

Otro obstáculo se asocia a la presencia mayoritaria de hombres en las juntas directivas, lo cual supone desventajas para las mujeres al momento de plantear ideas o proyectos. Algunas entrevistadas señalan que se sien- ten discriminadas e invisibilizadas por el directorio masculino. Cabe señalar que los cargos más importantes de las federaciones están cubiertos por hombres, principalmente la Presidencia.

«....sí, siento que hubo discriminación, sobre todo con la otra dirigente que en algún momento quiso ser Presidenta. Históricamente la presidencia de la federación ha estado en manos de los hombres». "...pero si no va secundada (opinión) a veces por la de otro hombre que, también diga que ella tiene razón, la opinión puede simplemente no ser considerada; entonces creo que eso es uno de los mayores obstáculos» (Amelia, 36 años, 1 hijo, divorciada).

«Por ejemplo, en la Federación Panamericana de... una vez se me dijo derechamente que yo era una aparecida en el... (deporte), que no sabía nada de... (deporte); y fue muy vinculado al tema de género. Sin embargo, los resultados han demostrado que no era así (Carolina, casada).

\section{Facilitadores}

$\mathrm{Si}$ bien, anteriormente se señaló que las funciones y roles familiares se sitúan como barreras relevantes para el acceso y participación en cargos directivos, las entrevistadas también han identificado a miembros de la familia como un importante apoyo para el desarrollo de sus funciones en la organización deportiva.

"....sí, bastante apoyo de mi familia, de mi marido principalmente que siempre ha tenido muy buena disposición porque yo a veces viajo y le dejo a mi hijo, de mi madre también..." (Camila, 58 años, 1 hijo, casada).

«... en mi familia nadie me ha planteado problemas respecto a que dedico más tiempo a una u otra cosa, pero tiene que ver con la forma que tenemos como familia de ver la vida, ya que no tenemos roles de trabajo doméstico establecidos... la verdad es que ningún problema» (Marta, 41 años, 1 hijo, casada).

"...el apoyo se basa en que me pueda ausentar y viajar periódicamente, y también gastar recursos familiares para solventar algunas cosas que no se pueden cubrir con fondos de la federación» (Daniela, 35 años, 0 hijo, casada).

Otro facilitador es contar con estudios de nivel superior (profesión) y desarrollar labores profesionales en contextos no deportivos. Para algunas entrevistadas la experiencia profesional en áreas distintas al deporte les permite aplicar esos conocimientos a la gestión de la federación.

"Necesitamos tener un piso (formación profesional) para que no se nos critique para acceder a ciertos puestos» (Marta, 41 años, 1 hijo, casada).

"...me ayudó mucho lo de Administración de Empresas. Antes había estudiado secretariado») (Camila, 58 años, 1 hijo, 
casada).

Otro elemento que no está necesariamente asociado al entorno social, pero que las entrevistadas plantean como relevante es el tipo de personalidad que poseen y que proyectan en las gestiones y desarrollo de las federaciones.

"...mi personalidad ha ayudado bastante en la toma de decisiones. Al principio se consideraba un puesto decorativo (Secretaria), pero posteriormente me posicioné en el puesto cambiando la mirada» (Daniela, 35 años, 0 hijo, casada).

"La verdad es que igual tengo una personalidad súper fuerte, entonces las personas codifican bien y saben quién puede también golpear más la mesa, pero la verdad creo que tenemos una relación de bastante respeto dentro del directorio ... (Marta, 41 años, 1 hijo, casada).

Otro facilitador corresponde a la capacitación en gestión deportiva, la cual es considerada como primordial por las entrevistadas. Algunas han tenido la oportunidad de realizar cursos de capacitación en gestión deportiva a través del Comité Olímpico de Chile o del Instituto Nacional de Deportes.

"...hice el diplomado MOSO (Managing Olympic Sport Organisations) que se otorga a través del COCH y Solidaridad Olímpica, postulé a la beca, la obtuve y el 2015 hice ese diplomado, lo cual me ha ayudado en mi desempeño dirigencial» (Carolina, casada).

\section{Estrategias}

A las entrevistadas se les consultó acerca de las estrategias o acciones que permitirían mejorar el acceso a cargos directivos y también desarrollar sus funciones adecuadamente en las federaciones. Entre los aspectos más destacados surge la necesidad de generar un cambio cultural y educativo en la sociedad, y en particular en los dirigentes masculinos.

"... yo creo en la necesidad de un cambio de mentalidad en la sociedad, porque esto no es algo que veamos sólo en el deporte; es en general. La mujer ha estado ahí, "pegando codazos" para ser visualizadas, el deporte también estuvo y todavía lo está... entonces es un poco de cambiar la mentalidad de todos y empezar a tener un poco de mayor visión; principalmente en los hombres. Tengo la esperanza puesta en las futuras generaciones» (Amelia, 36 años, 1 hijo, divorciada).

«... los hombres también tienen que aprender a trabajar con las mujeres. Yo creo que la gente más joven eso lo está aprendiendo ahora, ya que lo tienen como más asimilado» (Camila, 58 años, 1 hijo, casada).

"...esto tiene que ser sobre todo con mucha educación y de largo tiempo... tienes que trabajar preferentemente con los hombres para que entiendan y empaticen con la realidad de las mujeres» (Carolina, casada).

En línea con lo anterior, y de forma más específica, otra estrategia es la promoción de la educación y cultura en aspectos de género al interior de las federaciones, y en el deporte en general, esto significa educar en aspectos asociados al respeto, equidad e igualdad.

"...hay que partir por el tema de educar en equidad, la igualdad de oportunidades al interior de la federación, partiendo de esa base, se siente que en la federación las mujeres no son discriminadas» (Daniela, 35 años, 0 hijo, casada).

Un elemento recurrente y pragmático se relaciona al establecimiento de cuotas de participación por género en cargos directivos, las cuales deberían estar incorporadas en las normativas y estatutos de las federaciones.

"...es necesario aumentar la cuota de dirigentes mujeres a un 50/50 o un 60/40. Yo creo que puede haber una cuota desde la legalidad. Efectivamente dejarlo como una política pública, instaurarlo como algo legal dentro de las federaciones; la participación sí o sí de mujeres para que sea algo reglamentado y no como algo optativo» (Fernanda, 35 años, 3 hijos, divorciada).

Las entrevistadas también señalan que la capacitación en gestión deportiva representa una importante estrategia, ya que las empodera y prepara para asumir cargos de responsabilidad en las organizaciones.

"... todo lo que se está haciendo ahora, cursos de liderazgo femenino y de gestión, de entregar herramientas, de capacitarnos, son muy buenos y además invita a las mujeres a participar» (Camila, 58 años, 1 hijo, casada).

\section{Discusión}

La información indica que la falta de tiempo producto de las demandas familiares (Hinojosa-Alcalde et al., 2018; Moragas, 2014; Pérez et al., 2018), los prejuicios sobre el liderazgo y la capacidad femenina (Puig, 2007) y, la conformación mayoritariamente masculina de las juntas directivas (reproducción homosocial) (Kanter (1977), da como resultado una baja participación de las mujeres en estas instancias de las federaciones. Se suma a ello que, los cargos que usualmente cubren las mujeres son los que tienen menor poder de decisión o menor «peso» dentro de la junta directiva (Puig, 2007). Estas dificultades podrían asociarse según Moragas (2014), a los estereotipos de género imperantes, de acuerdo con la prescripción asociada al género femenino en particular, y a los comportamientos que se le asocian, donde los roles familiares dificultarían el desarrollo de otro tipo de actividades que no sean las del 
hogar. En este sentido, se podría indicar también la presencia de un hábito o habitus (Bourdieu, 2000) de acuerdo con los roles que deben cumplir hombres y mujeres, lo que a su vez tendría relación con la presencia de un «Régimen de Género» (Connell, 2009), lo cual se refleja en las respuestas de las entrevistadas.

Martínez (2017) indica que el habitus se produce debido a la posición de la persona en la estructura social, generando prácticas sociales estructuradas; además Pierre Bourdieu (2000) indica que existen agentes socializadores que contribuyen a delimitar el papel de las mujeres dentro de la sociedad, señalando que las actividades importantes o de honor son reservadas para los hombres, mientras que las actividades menos relevantes se reservan para las mujeres. Estos estereotipos de género impuestos mediante agentes socializadores podrían estar influyendo de forma consciente o inconsciente en las elecciones de hombres para los puestos directivos más importantes y afectando el desarrollo de las mujeres en cargos directivos.

Estas ideas preconcebidas de roles asignados a la persona en base a su género serían la razón que explica la presencia de la Reproducción Homosocial (Kanter, 1977), donde las cúpulas de las juntas directivas están conformadas casi exclusivamente por hombres, eligiendo estos de forma consciente o inconsciente a hombres similares a ellos. Asimismo, la Reproducción Homosocial y el Régimen de Género (el cual es impuesto por la sociedad a los individuos) podrían ser determinantes en la existencia de un Techo de Cristal (Hymowitz \& Schelhardt, 1986) en las federaciones deportivas chilenas, donde mujeres dirigentes que cuentan con las aptitudes y competencias técnicas, no son consideradas para los puestos más importantes o son designadas sólo a cargos simbólicos o complementarios, sin considerar el aporte que podrían realizar a la gestión deportiva.

Sobre los elementos que se reconocen como facilitadores, surgen de forma recurrente, y quizás con cierta contradicción, de acuerdo con las barreras identificadas, algunos factores asociados a la familia, ya que las entrevistadas señalan que el contar con el apoyo de la pareja (corresponsabilidad) y otros integrantes de la familia, les permite tener las condiciones necesarias de participación (tiempo y recursos), es decir, lograr una conciliación entre la vida familiar y las funciones directivas en la federación. Según la literatura, estas situaciones se producen principalmente en parejas jóvenes, pero de forma lenta (Hernández \& Lara, 2015).

Contar con una formación profesional y tener experiencia en gestión, se identifican como facilitadores, puesto que las capacidades técnicas de áreas como la administración de empresas o el conocimiento e interpretación de las leyes les permiten desenvolverse mejor en la dirección deportiva. Terjesen et al. (2009) manifiestan que la gobernanza corporativa mejora cuando las mujeres son incluidas en las juntas directivas de las empresas, pues contribuyen con su talento a las discusiones y decisiones; así también, Nielsen y Huse (2010) indican que la proporción de mujeres directoras se relaciona positivamente con la efectividad de la junta y el control estratégico. El avanzar hacia juntas directivas con mayor igualdad y equidad de género puede resultar beneficioso para el desempeño del directorio, tal como indican Hartarska y Nadolnyak (2012) quienes concluyen que la diversidad de género tiene un impacto positivo en la eficiencia organizacional.

Por otro lado, las dirigentes destacan que para desarrollar sus funciones directivas el poseer ciertas características personales como demostrar temperamento, liderazgo y pronunciarse sin temores, les facilita la instalación de ideas y la toma de decisiones. Esto muestra coherencia con los planteamientos de Bourdieu (2000) y de Burgess y Borgida (1999), quienes señalan que las mujeres para acceder a algunos puestos no solo deben cumplir con lo que exige explícitamente la descripción del cargo, sino que también deben poseer rasgos atribuidos tradicionalmente a los hombres tales como una voz fuerte o demostrar seguridad y agresividad. También se identifica como facilitador el poseer cursos o capacitaciones en gestión deportiva, lo que posicionaría positivamente a las dirigentes, permitiéndoles también profundizar en la administración de la federación y contribuir a la dirección. Además, estas instancias formativas les otorgan competencias técnicas que les permiten posicionarse de mejor forma frente a los demás directivos. Esto tiene concordancia con lo afirmado por Goslin y Kluka (2014), quienes indican que el participar de programas de educación sobre liderazgo deportivo afecta positivamente, tanto en la autoestima individual como a la voz colectiva de las mujeres dirigentes deportivas.

En cuanto a las estrategias, se identificó de forma recurrente, pero careciendo de una mayor precisión, la necesidad de un cambio cultural y educativo a nivel social y al interior de las federaciones, en el sentido de instalar la igualdad y equidad de género como parte de la cultura organizacional de estas instituciones. Cabe señalar que durante la VI Conferencia Mundial del International Working Group on Women \& Sport realizada en Helsinki en 2014 (IWG, 2014), se hizo hincapié en que se debe incorporar sistemáticamente la pers- 
pectiva de género a los programas de educación y capacitación de mujeres para cargos de liderazgo deportivo; además Ottesen et al. (2010) indican que la aplicación de cursos de educación y capacitación para mujeres en liderazgo deportivo ha sido una estrategia que ha dado buenos resultados en sus esfuerzos por disminuir la brecha de género en las organizaciones deportivas de países escandinavos.

Sobre lo señalado por las entrevistadas acerca de la adopción de cuotas de género como una forma de promover la inclusión de mujeres en las juntas deportivas, para Adriaanse y Schofield (2013) las cuotas se muestran como una herramienta muy útil para alcanzar la paridad de género, especialmente para lograr un número mínimo de mujeres integrantes de la junta. Sin embargo, se debe tener el cuidado de que el instaurar una cuota mínima de representación no se transforme en un simple requisito a cumplir; lo que según Rich et al. (2004) convertiría esta opción en un simple techo para ser alcanzado y que podría terminar por socavar el principio del mérito, haciendo pensar que las mujeres presentes en la junta directiva son sólo puestos decorativos incluidos para cumplir con un requisito impuesto. A lo anterior se suman la importancia de contar con acciones de capacitación que les permitan adoptar y emplear herramientas de gestión, fortaleciendo las competencias técnicas y su posicionamiento en las federaciones.

Finalmente, las entrevistadas relevan la necesidad de una mayor participación femenina en puestos directivos, dado que ello puede servir como modelo para otras mujeres interesadas en participar de la gestión deportiva. Esto es correspondiente con el planteamiento de Acosta y Carpenter (2014), puesto que exponen que las mujeres líderes se pueden transformar en modelos femeninos a seguir para otras mujeres, lo que podría contribuir a aumentar la presencia femenina en la dirección deportiva.

\section{Conclusiones}

Podemos señalar que se han identificado barreras que influyen y dificultan la participación femenina en las juntas directivas, siendo las más relevantes el desarrollo de las funciones o demandas familiares (conciliación con la vida familiar) de forma paralela con las actividades laborales, siendo el factor temporal (no contar con el tiempo requerido) el que permite agrupar estos elementos. Otras barreras se sitúan al interior de la junta directiva, donde las mujeres son estereotipadas, prejuiciadas y discriminadas por sus pares masculinos, lo que se refleja en que los puestos más importantes son cubiertos principalmente por hombres. A la vez, las propuestas, capacidades y opiniones femeninas son subestimadas y poco consideradas.

Entre los elementos facilitadores destacan el soporte proveniente de la familia; poseer una formación profesional y/o técnica y desempeñarse laboralmente en contextos no deportivos; poseer ciertas características en la personalidad de las dirigentes y, contar con capacitaciones que entreguen herramientas de gestión, también se sitúa como relevante.

Las estrategias que permitirían mejorar la participación de mujeres en cargos directivos de las federaciones serían: generar acciones educativas que promuevan cambios culturales al interior de las federaciones, en el sentido del reconocimiento de la equidad y la igualdad de género; aumentar normativamente o mediante leyes las cuotas de participación femenina en las juntas directivas de las federaciones; desarrollar y fortalecer programas de capacitación en liderazgo femenino que entreguen herramientas en el ámbito de la gestión deportiva.

Como limitaciones del trabajo se puede señalar en un principio el reducido número de entrevistas realizadas, sin embargo, esto se relaciona directamente con la baja presencia de mujeres en puestos directivos de las federaciones deportivas chilenas; además, se considera como limitación del trabajo, la inexistencia de estudios a nivel nacional que tomen como base la perspectiva de género y la participación en puestos directivos y de toma de decisiones en organizaciones deportivas chilenas, esto redujo la posibilidad de contrastación y discusión con evidencias asociadas de forma particular al contexto.

Los resultados de esta investigación relevan la necesidad de implementar desde las políticas públicas acciones que promuevan y concreten la igualdad y equidad de género en el sector deportivo.

\section{Agradecimientos}

Este artículo fue elaborado con la colaboración de UNESCO Guatemala, el Comité Olímpico Guatemalteco y la Confederación Deportiva Autónoma de Guatemala.

\section{Referencias}

Acosta, V. \& Carpenter, L. J. (2014). Women in Intercollegiate Sport:A Longitudinal, National Study, Thirty-FiveYear Update. 1977-2014. 
University of NewYork. Recuperado dehttps: / / eric.ed.gov/ ?id=ED570882

Adriaanse, J. (2019). Europe in world perspective. The Sydney Scoreboard Global Index for women in sport leadership. En Elling,A., Hovden, J. \& Knoppers,A (Eds.). Gender Diversity in European Sport Governance, 11-20. London: Routledge. p. 1120.

Adriaanse, J. \& Claringbould, I. (2016). Gender equality in sport leadership: From the Brighton Declaration to the Sydney Scoreboard. International Review for the Sociology of Sport, 51(5), 547-566. https://doi.org/10.1177/1012690214548493

Adriaanse, J. \& Schofield,T. (2013). Analysing gender dynamics in sport governance: A new regimes-based approach. Sport Management Review, 16(4), 498-513. https://doi.org/ 10.1016/j.smr.2013.01.006

Adriaanse, J. \& Schofield, T. (2014). The impact of gender quotas on gender equality in sport governance. Journal of Sport Management, 28(5), 485-497. https://doi.org/10.1123/ jsm.2013-0108

Álfaro, E. (2008). La participación de las mujeres en el deporte. Seminario Iberoamericano Mujer y Deporte. Seminario Iberoamericano Mujer y Deporte, Bolivia.

Ávila, R. \& Corvalán, M. (2016). La educación y la equidad de género: Limitaciones y alcances. En Ospina, H. F. \& RamírezLópez, C. A. (comps.), Pedagogía crítica latinoamericana y género. Bogotá, D. C.: Siglo del Hombre Editores; Manizales: Universidad de Manizales; Sabaneta: Cinde

Banks, M. (2010). Los datos visuales en Investigación cualitativa. Ediciones Morata.

Barberá, E., Ramos,A., Sarrió, M. \& Candela, C. (2002). Más allá del techo de cristal. Revista del Ministerio de Trabajo e Inmigración, 40. https: / /libros-revistas-derecho.vlex.es/vid/alla-techocristal-181887

Baumgartner, M. \& Schneider, D. (2010). Perceptions ofWomen in Management:AThematicAnalysis of Razing the Glass Ceiling. Journal of Career Development, 37(2), 559-576. https://doi.org/ $10.1177 / 0894845309352242$

Bourdieu, P. (2000). La Dominación Masculina. Anagrama.

Burgess, D. \& Borgida, E. (1999). Who women are, who women should be: Descriptive and prescriptive gender stereotyping in sex discrimination. Psychology, Public Policy, and Law, 5 (3), 665 692. https://doi.org/10.1037/1076-8971.5.3.665

Claringbould, I. \& Knoppers, A. (2008). Doing and Undoing Gender in Sport Governance. Sex Roles, 58(1), 81-92. https:/ /doi.org/10.1007/s11199-007-9351-9

Comité Olímpico de Chile. (2020). Federaciones - COCH. Recuperado de https://coch.cl/federaciones-coch/

Connell, R. (2009). Gender (2a ed.). Polity Press.

Creswell, J. (2014). Investigação qualitativa e projeto de pesquisa. Escolhendo entre cinco abordagens (3a ed.). Penso Editora.

De Lemus, S. \& Moya, M. (2004). Superando barreras: Creencias y aspectos motivacionales relacionados con el ascenso de las mujeres a puestos de poder. Revista de psicología general y aplicada, 57(2), 225-242.
Deloitte. (2019). Women in the boardroom. A global perspective. Recuperado de https://www2.deloitte.com/content/dam/ Deloitte/au/Documents/risk/deloitte-au-risk-women-inboardroom-sixth-edition.pdf

Flores, R. (2009). Observando observadores. Una introducción a las técnicas cualitativas de investigación social. Ediciones Universidad Católica de Chile.

Gaston, L., Blundell, M. \& Fletcher,T. (2020). Gender diversity in sport leadership: an investigation of United States of America National Governing Bodies of Sport. Managing Sport and Leisure, 25(6), 402-417. https://doi.org/10.1080/ 23750472.2020.1719189

GonzálezTirados, R. (2010). El problema de género en las estructuras empresariales españolas del Siglo XXI. ICE, Revista de Economía, 852, 113-125. http://www.revistasice.com/ index.php/ICE/article/view/1307

Goslin, A. \& Kluka, D. (2014). Women and sport leadership: Perceptions of Malawi women educated in sport business leadership. South African Journal for Research in Sport, Physical Education and Recreation, 36(3). https: / / repository.up.ac.za/ handle/2263/43377

Hartarska, V. \& Nadolnyak, D. (2012). Board size and diversity as governance mechanisms in community development loan funds in the USA. Applied Economics, 44(33), 4313-4329.https: / /doi.org/10.1080/00036846.2011.589812

Heinemann, K. (2008). Introducción a la metodología de la investigación empírica en las ciencias del deporte. Barcelona: Editorial Paidotribo.

Hernández, M. \& Lara, B. (2015). Responsabilidad familiar ¿una cuestión de género? RES. Revista de Educación Social, 21. https:/ /eduso.net/res/revista/21/el-tema-colaboraciones/responsabilidad-familiar-una-cuestion-de-genero

Hernández, R., Fernández, C. \& Baptista, M. (2014). Metodología de la investigación (6a ed.). McGraw-Hill / Interamericana Editores.

Hinojosa-Alcalde, I., Andrés, A., Serra, P., Vilanova,A., Soler, S. \& Norman, L. (2018). Understanding the gendered coaching workforce in Spanish sport. International Journal of Sports Science \& Coaching, 13, 4, 485-495. https://doi.org/ $10.1177 \% 2 \mathrm{~F} 1747954117747744$

Hymowitz, C. \& Schelhardt,T. (1986). The Glass-Ceiling:WhyWomen Can't Seem to Break the Invisible Barrier that Blocks Them from Top Jobs. TheWall Street Journal.

IWG. (2014). Helsinki exhorta al mundo del deporte a liderar el cambio, ser el cambio. International Working Group (IWG) on Women \& Sport. Recuperado de http://www.fisu.net/medias/ f $\begin{array}{llllllll}\mathrm{i} & \mathrm{C} & \mathrm{h} & \mathrm{i} & \mathrm{e} & \mathrm{r} & \mathrm{s}\end{array}$

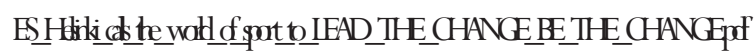

Kanter, R. (1977). Men andWomen of the Corporation. NewYork: Basic Books. Recuperado de https: / /www.hbs.edu/faculty/ Pages/item.aspx?num $=10807$

Lo Iacono, V., Symonds, P. \& Brown, D. (2016). Skype as a Tool for Qualitative Research Interviews. Sociological Research Online, 21(2), 103-117. https://doi.org/10.5153/sro.3952 
Martínez, J. (2017). El habitus. Una revisión analítica. Revista Internacional de Sociología, 75(3), e067-e067. https://doi.org/ 10.3989/ris.2017.75.3.15.115

Marugán, B. (2017). El deporte como bastión del machismo. Recreando imaginarios: Seminario internacional:Actas «Cine, deporte y género», 30-38. Recuperado de https: / / dialnet.unirioja.es/ servlet/articulo? codigo $=6421121$

Megheirkouni, M. (2014). Women-only leadership positions in the Middle East: exploring cultural attitudes towards Syrian Women for Sport Career development. Advancing Women in Leadership, 34, 64-78. https://doi.org/10.18738/ awl.v34i0.320

Menéndez, M. I. (2020). «You're made of what you do»: Impulso del deporte femenino a través de estrategias de femvertising en Nike. Retos, 38, 425-432. https://doi.org/10.47197/ retos.v38i38.76959

Monforte, J. \& Úbeda-Colomer, J. (2019). ‘Como una chica': Un estudio provocativo sobre estereotipos de género en educación física. Retos, 36, 74-79. https://doi.org/10.47197/ retos.v36i36.68598

Moragas, M. (2014). Les dones que presideixen els clubs esportius a Catalunya. Factors que incideixen en l'accés i en l'exercici del càrrec, i estil de lideratge [Tesis doctoral, Universitat Ramon Llull]. En TDXTesis Doctorals en Xarxa.http:/ / www.tdx.cat/handle/ 10803/145029

Nielsen, S. \& Huse, M. (2010). The Contribution of Women on Boards of Directors: Going beyond the Surface. Corporate Governance: An International Review, 18(2), 136-148. https:// doi.org/10.1111/j.1467-8683.2010.00784.x

Oakley, J. (2000). Gender-based Barriers to Senior Management Positions: Understanding the Scarcity of Female CEOs. Journal of Business Ethics, 27(4), 321-334. https:/ /doi.org/10.1023/ A:1006226129868

ONU. (s. f.). Igualdad de género: ¿por qué es importante? Recuperado de https://www.un.org/sustainabledevelopment/es/wpcontent/uploads/sites/3/2016/10/ 5_Spanish_Why_it_Matters.pdf

ONU. (2015). Igualdad de género y empoderamiento de la mujer. Desarrollo Sostenible. Recuperado de https://www.un.org/ sustainabledevelopment/es/gender-equality/

ONU Mujeres. (2021). Plan Estratégico para 2022-2025. Recuperado de https://undocs.org/es/UNW/2021/6

Ottesen, L., Skirstad, B., Pfister, G. \& Habermann, U. (2010). Gender relations in Scandinavian sport organizations - A comparison of the situation and the policies in Denmark, Norway and Sweden. Sport in Society, 13(4), 657-675. https: / /doi.org/10.1080/17430431003616423

Parzinger, M., Nath, R. \& Lemons, M. (2001). Examining the Effect of the Transformational Leader on Software Quality. Software Quality Journal, 9(4), 253-267. https://doi.org/ 10.1023/A:1013763119819

Pérez, M.,Vilanova,A. \& Soler, S. (2018). Mercado de trabajo en el deporte y género: un estudio comparativo entre las condiciones de trabajo de las tituladas y los titulados en Ciencias de la
Actividad Física y el Deporte por las universidades catalanas. Revista de Humanidades, 34, 195-216, 2018. http:// revistas.uned.es/index.php/rdh/article/view/19731/ 18120

Pfister, G. (2010). Are the women or the organisations to blame? Gender hierarchies in Danish sports organisations. International Journal of Sport Policy and Politics, 2(1), 1-23. https: / / doi.org/ $10.1080 / 19406941003634008$

Puig, N. (2007). Mujeres, puestos de decisión y organizaciones deportivas: Barreras y propuestas. En Diputación General de Aragón(Ed.). Actas de las Jornadassobre mujer y deporte. Zaragoza, España.

Rich, E., Henry, I.,White,A., Radzi,W., Shelton, C. \&Theodoraki, E. (2004). Women, leadership and the Olympic Movement: Research Report to the International Olympic Committee. Loughborough University. https://library.olympics.com/Default/doc/ SYRACUSE/57403/women-leadership-and-the-olympicmovement-final-report-january-2004-research-undertakenby-the-insti?_lg=en-GB

Ruiz Rabadán, S. \& Moya-Mata, I. (2020). Las deportistas olímpicas en los libros de texto de educación física: ¿presencia o ausencia de referentes en nuestro alumnado? Retos, 38, 229234. https: / / doi.org/10.47197/retos.v38i38.74833

Shaw, S. \& Hoeber, L. (2003). «A Strong Man Is Direct and a Direct Woman Is a Bitch»: Gendered Discourses andTheir Influence on Employment Roles in Sport Organizations. Journal of Sport Management, 17(4), 347-375. https://doi.org/10.1123/ jsm.17.4.347

Smith, M. \& Wrynn, A. (2013). Women in the 2012 Olympic and Paralympic games: An analysis of participation and leadership opportunities. SHARP Center forWomen and Girls. Recuperado de https://sharp.research.umich.edu/wp-content/ uploads/2017/03/olympic_report_2012_final-4-1113.pdf

Sullivan, J. (2013). Skype: An Appropriate Method of Data Collection for Qualitative Interviews? The Hilltop Review, 6(1). https: / /scholarworks.wmich.edu/hilltopreview/vol6/ iss $1 / 10$

Terjesen, S., Sealy, R., \& Singh, V. (2009). Women Directors on Corporate Boards: A Review and Research Agenda. Corporate Governance: An International Review, 17(3), 320-337. https:/ / doi.org/10.1111/j.1467-8683.2009.00742.x

Women in Sport. (2015). Checklist for change. Building a sustainable pipeline to gender diversity in sport leadership. Women in Sport. Recuperado de https://www.womeninsport.org/wpcontent/uploads / 2017/10/FINAL_Checklist-forchange_Trophy_Women_031115.pdf

Zanatta,T., Freitas, D., Carelli, F. \& Costa, I. (2018). El perfil del gestor deportivo brasileño: revisión bibliográfica sistemática. Movimento, 24(1), 291-304. https://doi.org/10.22456/ 1982-8918.73803 